\title{
Nucleon generalized form factors and sigma term from lattice QCD near the physical quark mass
}

\author{
G.S. Bali, S. Collins, B. Gläßle, M. Göckeler, J. Najjar, R. Rödl, A. Schäfer, R. Schiel, \\ W. Söldner, A. Sternbeck* and P. Wein \\ Institut für Theoretische Physik, Universität Regensburg, 93040 Regensburg, Germany \\ E-mail: andre.sternbeck@ur.de
}

\begin{abstract}
We present new $N_{f}=2$ data for the nucleon generalized form factors, varying volume, lattice spacing and pion mass, down to $150 \mathrm{MeV}$. We also give an update of our direct calculation of the nucleon sigma term for a range of pion mass values including the lightest one.
\end{abstract}

31st International Symposium on Lattice Field Theory - LATTICE 2013

July 29 - August 3, 2013

Mainz, Germany

\footnotetext{
* Speaker.
} 


\section{Introduction}

Lattice QCD calculations are an essential tool to study the inner structure of nucleons. Moments of parton distribution functions and form factors or, more recently, generalized form factors have always been popular targets in this regard. These observables provide important information on the distribution of momentum, spin and charge within a nucleon and are accessible on the lattice via expectation values of local operators, $O^{\mu_{1} \mu_{2}, \ldots}$. For example,

$$
\left\langle N\left(P^{\prime}\right)\left|O_{V}^{\mu v}\right| N(P)\right\rangle=\bar{U}\left(P^{\prime}\right)\left\{\gamma^{\{\mu} \bar{P}^{v\}} A_{20}(t)-\frac{i \sigma^{\rho\{\mu} \Delta_{\rho} \bar{P}^{v\}}}{2 m_{N}} B_{20}(t)+\frac{\Delta^{\{\mu} \Delta^{v\}}}{m_{N}} C_{2}(t)\right\} U(P)
$$

gives access to the twist- 2 generalized form factors $A_{20}, B_{20}$ and $C_{2}$ and also to the second (Mellin) moment of the nucleon's parton distribution $\langle x\rangle=A_{20}(0)$ (see, e.g., [1] for a comprehensive list).

Besides structure functions, sigma terms, $\sigma_{q}=m_{q}\langle N|\bar{q} q| N\rangle$ for the quark $q=\{u, d, s\}$, are also of interest. They parametrize the (small) contribution of quarks to the nucleon mass and are needed, e.g., for precision measurements of SM parameters or dark-matter searches. Experimentally, sigma terms are only indirectly accessible but lattice QCD provides a way to calculate them. In particular, the sigma term of the strange quark, $\sigma_{s}$, or the so-called pion-nucleon sigma term $\sigma_{\pi N}=\sigma_{u}+\sigma_{d}$ of the (approximate mass-degenerate) light quarks have been addressed on the lattice in the past [2-4]. Until recently it was however numerically too expensive to perform these calculations in the vicinity of the physical point or to directly evaluate $\sigma_{\pi N}$. Therefore, most lattice values of $\sigma_{\pi N}$ are from indirect determinations, for example, from chiral extrapolations of lattice data for the nucleon mass (via the Feynman-Hellmann theorem) [5, 6]. Often these extrapolations are performed up to relatively large pion masses $m_{\pi}$, which limits the precision of the final (physical) value.

Lattice determinations of structure functions suffer similar limitations, in addition to the problem of excited-state contributions which become more severe the closer one gets to the physical point. With the advance of new lattice techniques and ever more powerful computers the situation has, however, improved. Now lattice QCD calculations start to approach the physical point and excited-state contaminations can be removed more efficiently [7]. Here we will present new data for the generalized form factors and $\sigma_{\pi N}$, including an estimate almost at the physical point.

\section{Lattice setup}

Our lattice calculations are performed on configurations generated by the Regensburg group and QCDSF of $N_{f}=2$ nonperturbatively-improved Wilson fermions and the standard Wilson gauge action with $\beta=5.29$ and 5.40. The lattice spacings are 0.07 and $0.06 \mathrm{fm}$, respectively and our pion mass values range from $491 \mathrm{MeV}$ down to $150 \mathrm{MeV}$. Lattice sizes are chosen accordingly: $L m_{\pi} \geq$ 3.4 or higher (see Table 1 for details). For the translation of our lattice results into physical units we assume $r_{0}=0.5 \mathrm{fm} \mathrm{[6]} \mathrm{and} \mathrm{use} \mathrm{nonperturbative} \mathrm{renormalization} \mathrm{constants} \mathrm{for} \mathrm{the} \mathrm{conversion} \mathrm{of}$ the form factors to the $\overline{\mathrm{MS}}$ scheme (renormalization scale $\mu=2 \mathrm{GeV}$ ) [8].

Our calculations feature an optimized source and sink smearing to reduce the impact of excitedstate contaminations. These sources and sinks are created using typically 300-400 steps of Wuppertal smearing and APE-smeared spatial links. Two or three sources are used per configuration, 


\begin{tabular}{cccrcccc}
\hline \hline$\beta$ & $\kappa$ & lattice & $N \times M$ & $a[\mathrm{fm}]$ & $m_{\pi}[\mathrm{MeV}]$ & $L m_{\pi}$ & $t_{\text {sink }} / \mathrm{a}$ \\
\hline 5.29 & 0.13620 & $24^{3} \times 48$ & $1124 \times 2$ & 0.07 & 430 & 3.7 & 15 \\
& 0.13632 & $32^{3} \times 64$ & $2027 \times 2$ & & 294 & 3.4 & 15 \\
& 0.13632 & $40^{3} \times 64$ & $2028 \times 2$ & & 289 & 4.2 & 15 \\
& 0.13640 & $64^{3} \times 64$ & $940 \times 3$ & & 150 & 3.5 & 15 \\
5.40 & 0.13640 & $32^{3} \times 64$ & $1170 \times 2$ & 0.06 & 491 & 4.8 & 17 \\
& 0.13660 & $48^{3} \times 64$ & $2178 \times 2$ & & 260 & 3.8 & 17 \\
\hline
\end{tabular}

Table 1: Simulation parameters. $N$ is the number of configurations and $M$ of sources per configuration.

depending on the ensemble parameters (see Table 1). The first source is placed at a random site, the remaining one (or two) such that the distance between them is maximized.

The generalized form factors (GFFs) are extracted from fits to ratios

$$
R\left(t_{\text {sink }}, \tau, p^{\prime}, p\right)=\frac{C_{3 p t}^{\mathscr{O}}\left(t_{\text {sink }}, \tau, \vec{p}^{\prime}, \vec{p}\right)}{C_{2 p t}\left(t_{\text {sink }}, \vec{p}^{\prime}\right)} \sqrt{\frac{C_{2 p t}\left(t_{\text {sink }}-\tau, \vec{p}\right) C_{2 p t}\left(\tau, \vec{p}^{\prime}\right) C_{2 p t}\left(t_{\text {sink }}, \vec{p}^{\prime}\right)}{C_{2 p t}\left(t_{\text {sink }}-\tau, \vec{p}^{\prime}\right) C_{2 p t}(\tau, \vec{p}) C_{2 p t}\left(t_{\text {sink }}, \vec{p}\right)}}
$$

of nucleon two and three-point functions, $C_{2 p t}(t, \vec{p})$ and $C_{3 p t}^{\mathscr{O}}\left(t_{\operatorname{sink}}, \tau, \vec{p}^{\prime}, \vec{p}\right)$. For the calculation of the latter we use the standard sequential-source technique with a current inserted at $0 \ll \tau \ll t_{\text {sink }}$ (see [9] for our calculation using stochastic noise). $t_{\text {sink }}$ denotes the source-sink separation. We find that $t_{\operatorname{sink}}=15$ and $t_{\operatorname{sink}}=17$ (in lattice units) are sufficient for our calculations at $\beta=5.29$ and $\beta=5.40$, respectively. For tests using different $t_{\text {sink }}$ values see [7]. We restrict ourselves to the isovector case, but disconnected contributions are in progress.

For the scalar forward matrix element we have already preliminary data, including disconnected contributions. We extend our former analysis of $\sigma_{\pi N}$ [3] to a range of pion mass values. The scalar matrix elements $\langle N|\bar{q} q| N\rangle$, from which we obtain estimates for $\sigma_{\pi N}$, are extracted from ratios of three- and two-point functions at zero lattice momentum and with a stochastic estimator for the disconnected contributions.

\section{Results for Generalized Form Factors}

We start the discussion of results with the GFFs, namely the second (Mellin) moments of the vector and axial-vector nucleon GPDs. ${ }^{1}$ The relevant form factors are $A_{20}, B_{20}$ and $C_{2}$ for the vector GPDs, and $\tilde{A}_{20}$ and $\tilde{B}_{20}$ for the axial-vector GPDs. Preliminary data for $A_{20}, B_{20}$ and $C_{2}$ were already shown in [10]. Here we provide an update for these (obtained with our improved sink-source smearing) and also present results from a new gauge ensemble at almost physical quark $\operatorname{mass}\left(m_{\pi} \approx 150 \mathrm{MeV}\right)$.

A comparison of new and old points is shown in Fig. 1 (left) for the example of $A_{20, u-d}^{\overline{\mathrm{MS}}}$. Our new points lie systematically below the old points and this difference is almost independent of the momentum transfer $Q^{2}$, and roughly of the same magnitude as we see for $\langle x\rangle_{u-d}=A_{20, u-d}^{\overline{\mathrm{MS}}}(0)$ (see [7]). Since all data sets originate from the same gauge ensembles $\left(m_{\pi} \approx 289 \mathrm{MeV}\right)$, but a different

\footnotetext{
${ }^{1}$ Results for the first and third moments will be published elsewhere. Pion structure function results from the same gauge ensembles can be found in [11].
} 

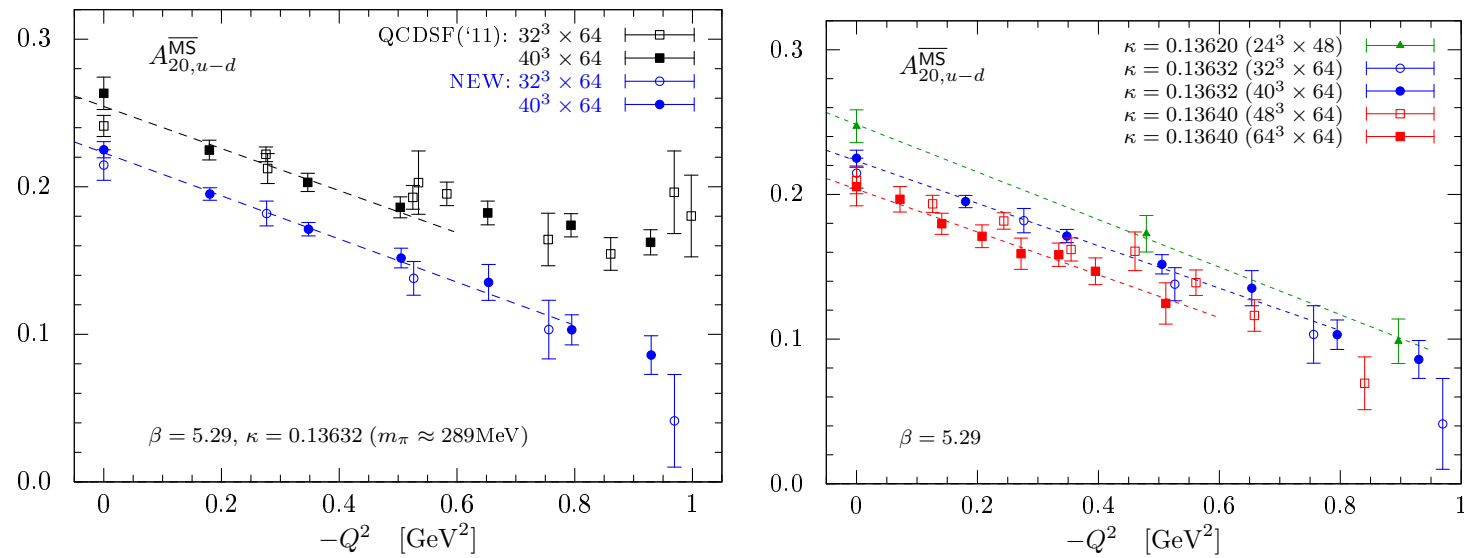

Figure 1: $A_{20, u-d}^{\overline{\mathrm{MS}}}$ versus $-Q^{2}$. Left: comparison of two types of source smearing for fixed lattice parameters $(\beta=5.29, \kappa=0.13632)$. Black squares are for Jacobi smearing (old data from [10]), blue circles for the improved smearing. Right: $Q^{2}$-dependence for three pion masses: $m_{\pi}=151$ (red squares), 289 (blue circles) and $429 \mathrm{MeV}$ (green diamonds). The points are all for the improved smearing and $\beta=5.29$.
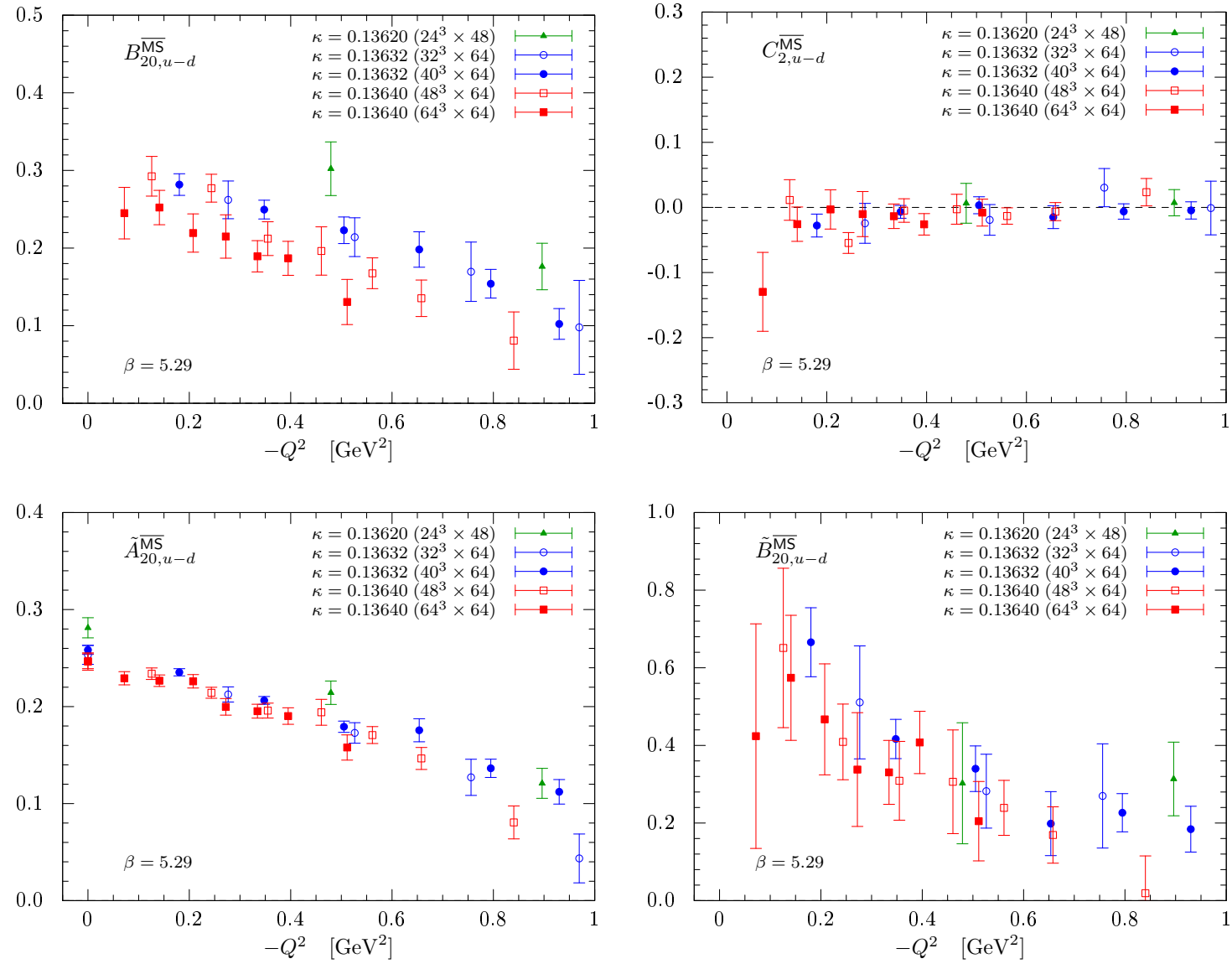

Figure 2: As Fig. 1 (right) but for $B_{20, u-d}^{\overline{\mathrm{MS}}}$ (top left), $C_{2, u-d}^{\overline{\mathrm{MS}}}$ (top right), $\tilde{A}_{20, u-d}^{\overline{\mathrm{MS}}}$ (bottom left) and $\tilde{B}_{20, u-d}^{\overline{\mathrm{MS}}}$ (bottom right). Note that all data are still preliminary. 
type of sink-source smearing was used, we believe our improved smearing is the main reason for the down-shift of our new points, as has been discussed in detail in [7].

Deviations are also seen for $B_{20, u-d}$, but here these deviations decreases with $\left|Q^{2}\right| \rightarrow 0$. In fact, the slopes of the new and old points differ, but towards $Q^{2}=0$ the points tend to the same values. For $C_{20, u-d}$ we practically see no difference between old and new points.

Looking at the $m_{\pi}$-dependence, we see this dependence is now more pronounced than for the old data. For example, for $A_{20, u-d}\left(Q^{2}\right)$ we see that points for $Q^{2}<0$ differ for different pion masses, while in [10] we saw this difference to decrease with $Q^{2} \rightarrow 0$ (cf. Fig. 2 of [10] to Fig. 1 (right)). Also for $B_{20, u-d}$ and $\tilde{A}_{20}$ we observe a vertical separation of points for different pion masses that does not disappear as $Q^{2} \rightarrow 0$.

When the full statistics is reached we should be able to provide precise data for the low- $Q^{2}$ dependence of the GFFs. We will also perform combined fits of these five form factors to new (full) 1-loop expressions from Baryon Chiral perturbation theory [12]. As mentioned, calculations including disconnected contributions are in progress.

\section{Results for the Nucleon Sigma term}

For the scalar matrix element we already have some preliminary results which include contributions from disconnected diagrams. This allows us to update our former data on $\sigma_{\pi N}$. Our new $\sigma_{\pi N}$ data are shown in Fig. 3 for pion masses between $150 \mathrm{MeV}$ and $491 \mathrm{MeV}$. There we also show our previous estimate at $m_{\pi} \approx 290 \mathrm{MeV}$ [3] and the $N_{f}=2+1+1$ point from the ETM collaboration at $m_{\pi} \approx 390 \mathrm{MeV}$ [4]. Both agree within errors with our new $\left(N_{f}=2\right)$ points.

As mentioned above, at the physical point one knows $\sigma_{\pi N}^{\text {phys }}$ only indirectly, for example from chiral extrapolations of lattice data for the nucleon mass. Recent values for $\sigma_{\pi N}^{\text {phys }}$ from such studies range between 32 and $52 \mathrm{MeV}[5,6]$. Our new direct (but still preliminary) data point at $m_{\pi} \approx$ $150 \mathrm{MeV}(a \approx 0.07 \mathrm{fm})$ lies in the lower half of that range (see Fig. 3).

With these new points for $\sigma_{\pi N}$ we can also refine our scale setting of $r_{0}$ for which in [6] we used nucleon mass data for a range of pion mass values below $500 \mathrm{MeV}$ and the sigma-term at $m_{\pi} \approx$ $290 \mathrm{MeV}$ and fitted them simultaneously to the chiral expressions for $\sigma_{\pi N}\left(m_{\pi}^{2}\right)$ and $M_{N}\left(m_{\pi}^{2}\right)$. Since $\sigma_{\pi N}\left(m_{\pi}^{2}\right)$ is related to the slope of $M_{N}$, this particular combination helps to reduce the uncertainties of the chiral fits and hence of the scale setting. In [6] we had $\sigma_{\pi N}$ only for a single $m_{\pi}$, but now we have both $\sigma_{\pi N}\left(m_{\pi}^{2}\right)$ and $M_{N}\left(m_{\pi}^{2}\right)$ for all $m_{\pi}$.

An example for such a simultaneous fit (which also includes volume corrections) is shown in Fig. 4. There, the (blue) slopes are given through the $\sigma_{\pi N}\left(m_{\pi}^{2}\right)$ data and the (red) squares are the finite-volume corrected data points for the nucleon mass. This fit is still preliminary and only shown for a particular pion mass range, but it demonstrates the potential of our approach.

Note also the physical point marked by a (green) full circle in Fig. 4. For this we use our estimate for $r_{0}$ from [6], which is clearly supported by our new data. The fit was not forced to go through the physical point.

\section{Conclusions}

We have reported on our reanalysis of the nucleon generalized form factors (GFFs) on $N_{f}=2$ 


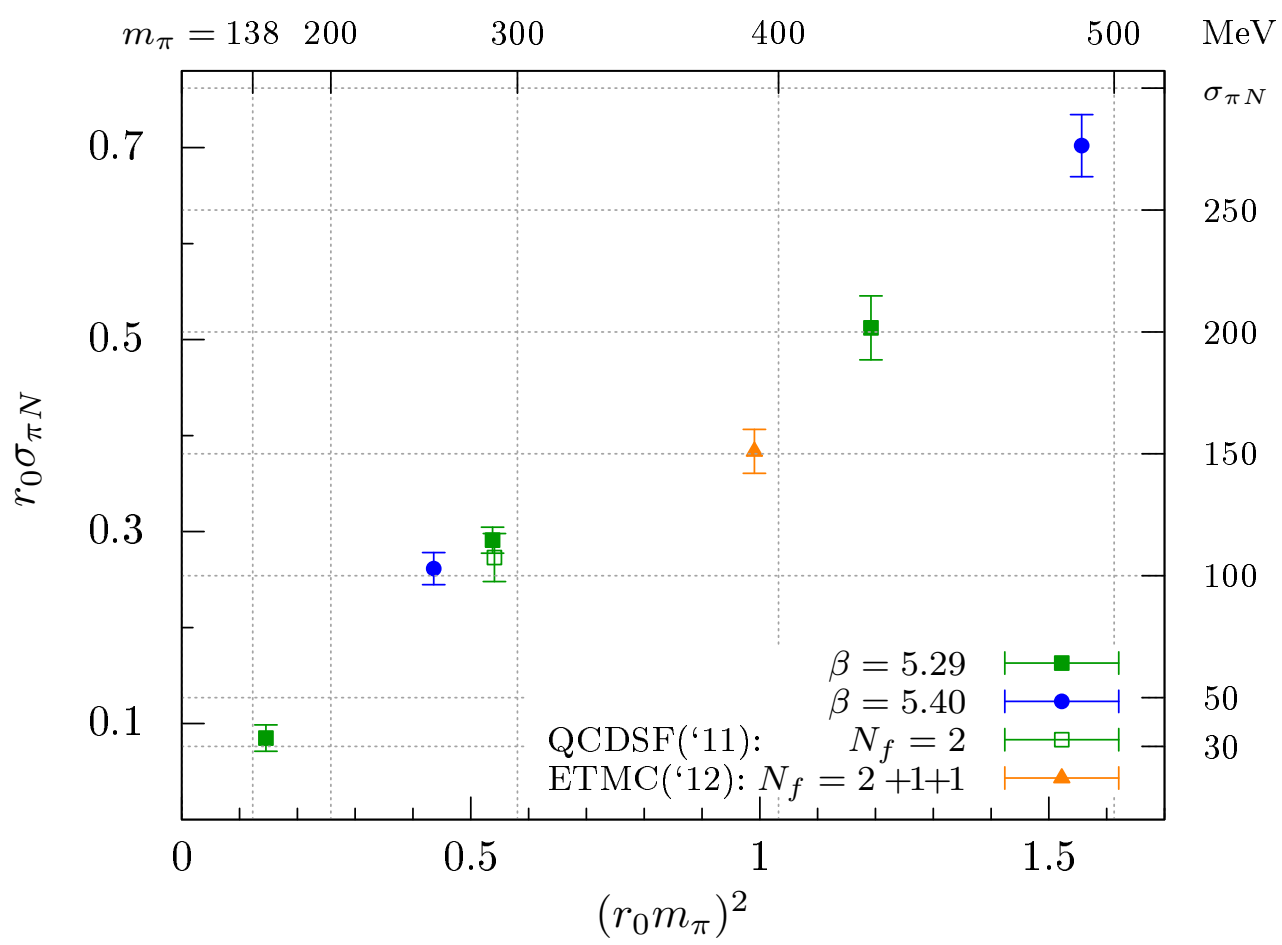

Figure 3: The $N_{f}=2$ pion-nucleon sigma term versus $m_{\pi}^{2}$. The ETMC point is from [4] and the QCDSF point from [3]. The right $\mathrm{y}$-axis and top $\mathrm{x}$-axis give the corresponding physical numbers.

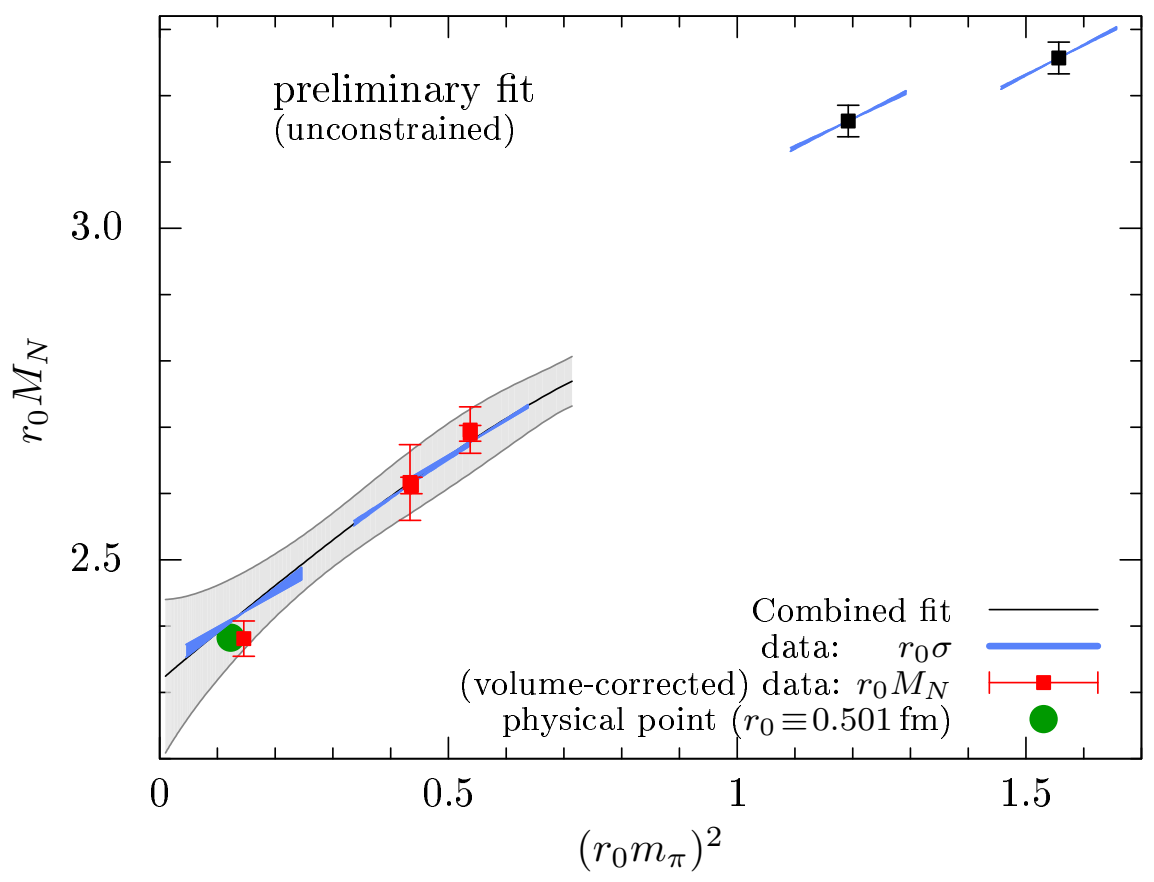

Figure 4: Combined fit to the (volume-corrected) nucleon mass data (red squares) and sigma term data (blue slopes), similar to what we did in [6]. The physical point is marked by a green circle assuming $r_{0}=0.501 \mathrm{fm}$ [6]. The fit was not "constrained" to go through the physical point. 
gauge field ensembles and added a new ensemble with $m_{\pi} \approx 150 \mathrm{MeV}$. Our calculations feature an optimized source and sink smearing for which we find excited-state contaminations are much reduced (see also [7]). A comparison of our new and old data for the GFFs shows a better removal of excited state contributions that changes the $Q^{2}$ dependence. In particular the $m_{\pi}$-dependence is much more pronounced.

Along with this re-calculation of the GFFs, we have also extended our calculation of $\sigma_{\pi N}$. We have provided here new $N_{f}=2$ data for $\sigma_{\pi N}\left(m_{\pi}\right)$ for pion masses from $m_{\pi} \approx 491 \mathrm{MeV}$ down to $m_{\pi} \approx 150 \mathrm{MeV}$, which will allow us to give an improved estimate of $\sigma_{\pi N}^{\text {phys }}$ in the near future.

The presented results should be considered as still preliminary. A complete analysis will follow as soon as the full statistics has been reached for all gauge ensembles.

Numerical calculations have been performed on the SuperMUC system at the LRZ/Germany and the FERMI BG/Q machine at CINECA/Italy. We acknowledge PRACE (project 2011050791) for awarding us access to the FERMI BG/Q machine. We have made use of the Chroma software suite [13] adapted for our needs. For the generation of gauge field configurations we used QPACE and the BQCD software [14] including an improved inverter [15]. This work has been supported in part by the DFG (SFB/TR 55, Hadron Physics from Lattice QCD) and the EU under grant 238353 (ITN STRONGnet). A.St acknowledges support by the European Reintegration Grant (FP7-PEOPLE-2009-RG, No.256594).

\section{References}

[1] P. Hägler, Phys.Rept. 490, 49 (2010), arXiv:0912.5483 [hep-lat].

[2] R. Babich et al., Phys.Rev. D85, 054510 (2012), arXiv:1012.0562 [hep-lat].

[3] G. S. Bali et al., Phys.Rev. D85, 054502 (2012), arXiv:1111.1600 [hep-lat].

[4] S. Dinter et al. (ETM Collaboration), JHEP 1208, 037 (2012), arXiv:1202.1480 [hep-lat].

[5] R. Young and A. Thomas, Phys.Rev. D81, 014503 (2010), arXiv:0901.3310 [heplat]; R. Horsley et al. (QCDSF-UKQCD Collaborations), Phys.Rev. D85, 034506 (2012), arXiv:1110.4971 [hep-lat]; S. Dürr et al., Phys.Rev. D85, 014509 (2012), arXiv:1109.4265 [hep-lat]; P. Shanahan, A. Thomas, and R. Young, Phys.Rev. D87, 074503 (2013), arXiv:1205.5365 [nucl-th]; A. Chowdhury et al., Nucl.Phys. B871, 82 (2013), arXiv:1212.0717 [hep-lat]; L. Alvarez-Ruso, T. Ledwig, J. Martin Camalich, and M. VicenteVacas, Phys.Rev. D88, 054507 (2013), arXiv:1304.0483 [hep-ph].

[6] G. Bali et al., Nucl.Phys. B866, 1 (2013), arXiv:1206.7034 [hep-lat].

[7] G. Bali et al., PoS Lattice 2013, 290 (2013), arXiv:1311.7041 [hep-lat].

[8] M. Göckeler et al., Phys.Rev. D82, 114511 (2010), arXiv:1003.5756 [hep-lat].

[9] G. S. Bali et al.(2013), arXiv:1311.1718 [hep-lat].

[10] A. Sternbeck et al., PoS LATTICE2011, 177 (2011), arXiv:1203.6579 [hep-lat].

[11] G. Bali et al., PoS LATTICE 2013, 447 (2013), arXiv:1311.7639 [hep-lat].

[12] P. Wein et al., work in progress.

[13] R. G. Edwards and B. Joo (SciDAC + LHPC + UKQCD), Nucl.Phys.Proc.Suppl. 140, 832 (2005), arXiv:hep-lat/0409003 [hep-lat].

[14] Y. Nakamura and H. Stüben, PoS LATTICE2010, 040 (2010), arXiv:1011.0199 [hep-lat].

[15] A. Nobile, PoS LATTICE2010, 034 (2010), arXiv:1109.4279 [hep-lat] 